\title{
DNA-binding-dependent androgen receptor signaling contributes to gender differences and has physiological actions in males and females
}

\author{
Helen E MacLean ${ }^{1}$, Alison J Moore ${ }^{2}$, Stephen A Sastra ${ }^{1}$, Howard A Morris ${ }^{2}$, Ali Ghasem-Zadeh ${ }^{1,3}$, \\ Kesha Rana ${ }^{1}$, Anna-Maree Axell ${ }^{1}$, Amanda J Notini ${ }^{1}$, David J Handelsman ${ }^{4}$, Ego Seeman ${ }^{1,3}$, \\ Jeffrey D Zajac ${ }^{1}$ and Rachel A Davey ${ }^{1}$ \\ ${ }^{1}$ Department of Medicine, University of Melbourne, Austin Health, Heidelberg, Victoria 3084, Australia \\ ${ }^{2}$ Hanson Institute, Adelaide, South Australia 5000, Australia \\ ${ }^{3}$ Department of Endocrinology, Austin Health, Heidelberg, Victoria 3084, Australia \\ ${ }^{4}$ ANZAC Research Institute, University of Sydney, Concord, New South Wales 2139, Australia \\ (Correspondence should be addressed to H E MacLean; Email: hmaclean@unimelb.edu.au)
}

\begin{abstract}
We used our genomic androgen receptor (AR) knockout (ARKO) mouse model, in which the AR is unable to bind DNA to: 1) document gender differences between males and females; 2) identify the genomic (DNA-binding-dependent) AR-mediated actions in males; 3) determine the contribution of genomic AR-mediated actions to these gender differences; and 4) identify physiological genomic AR-mediated actions in females. At 9 weeks of age, control males had higher body, heart and kidney mass, lower spleen mass, and longer and larger bones compared to control females. Compared to control males, ARKO males had lower body and kidney mass, higher splenic mass, and reductions in cortical and trabecular bone. Deletion of the AR in ARKO males abolished the gender differences in heart and cortical bone.
\end{abstract}

Compared with control females, ARKO females had normal body weight, but $14 \%$ lower heart mass and heart weight/ body weight ratio. Relative kidney mass was also reduced, and relative spleen mass was increased. ARKO females had a significant reduction in cortical bone growth and changes in trabecular architecture, although with no net change in trabecular bone volume. In conclusion, we have shown that androgens acting via the genomic AR signaling pathway mediate, at least in part, the gender differences in body mass, heart, kidney, spleen, and bone, and play a physiological role in the regulation of cardiac, kidney and splenic size, cortical bone growth, and trabecular bone architecture in females.

Journal of Endocrinology (2010) 206, 93-103

\section{Introduction}

Males and females show sexual dimorphism in a number of tissues. Males tend to be taller, with higher muscle and bone mass and lower fat mass than females, with differences also observed in the immune system, nervous system, and in erythropoiesis (Mooradian et al. 1987). These sex differences are likely to be due to differences in the concentrations of estrogen and testosterone as well as the expression of their receptors. Androgens, predominantly testosterone and dihydrotestosterone (DHT), have traditionally been thought of as the male sex hormones, which are essential for masculinization of the developing male fetus and development of secondary sexual characteristics at puberty. The non-reproductive actions of androgens in males have been investigated more recently, particularly the anabolic actions of androgens in the musculoskeletal system. Both skeletal muscle mass and bone mineral density are androgen responsive, as demonstrated in studies in human subjects (Behre et al. 1997, Chen et al. 2005) and mouse models (Wakley et al. 1991, Axell et al. 2006).

The classic paradigm of androgen action is that androgens act via the androgen receptor (AR), a ligand-dependent nuclear transcription factor (MacLean et al. 1997). This view is supported by the fact that loss of function mutations in the AR gene causes androgen insensitivity syndrome (AIS), in which affected males are androgen resistant and have a female external phenotype (MacLean et al. 2004). However, more recently, it has become apparent that the actions of androgens are more complex than this simple model suggests. It is now clear that in males, some androgen actions also arise through aromatization of androgens to estrogens and activation of 
the estrogen receptor (ER). This is evidenced by the fact that men with ER or aromatase gene mutations lack the pubertal growth spurt, fail to fuse their epiphyses, and have osteopenia (Smith et al. 1994, Grumbach \& Auchus 1999). Similarly, male mice with targeted deletion of the aromatase gene show loss of male-type sexual behaviors, increased adiposity, reduced bone length, and mineralization (Jones et al. 2000, Oz et al. 2001, Robertson et al. 2001).

In addition to the transcriptional actions of the AR, non-DNA-binding-dependent pathways have been identified in vitro (Kousteni et al. 2001, Estrada et al. 2003, Gill et al. 2004, Kang et al. 2004, Norris et al. 2009). The physiological relevance of this AR signaling is unclear, as previous studies in AR-null knockout (ARKO) mouse models have not distinguished between DNA-binding-dependent and -independent actions of the AR (Yeh et al. 2002, Kawano et al. 2003, De Gendt et al. 2004).

In addition to the ER-dependent actions of androgens in males, the potential physiological actions of androgens in females are also being identified. The pharmacological effects of exogenous androgens in females range from hirsutism and masculinization to muscle and bone anabolism (Strauss et al. 1985, Buchanan et al. 1988), and pathological effects of excess androgen occur in polycystic ovarian syndrome (Nisenblat \& Norman 2009). Human bone cells from male and female donors have similar concentrations of ARs, suggesting that androgens may play an important role in the maintenance of bone mass in both sexes (Colvard et al. 1989). Further supportive evidence for a role of the AR in females is provided by analyses of the reproductive system of ARKO female mice demonstrating a physiological role for androgens in ovarian folliculogenesis, uterine development, and mammary gland development (Yeh et al. 2003, Hu et al. 2004, Shiina et al. 2006, Walters et al. 2007, 2009). Other physiological actions of androgens in females have yet to be identified.

Since males and females express the AR at similar levels in many tissues (Rinn et al. 2004), but the production rate of testosterone is 20-30-fold higher in men (Handelsman 2010), we hypothesize that phenotypic differences between males and females are attributed at least in part to androgen actions via the genomic AR pathway. Furthermore, we also propose that androgens acting via the genomic AR signaling pathway play an important physiological role in non-reproductive tissues of both males and females. In this study, we have utilized our global ARKO mice, which have an in-frame deletion of the second zinc finger of the DNA-binding domain that abolishes genomic AR signaling (Notini et al. 2005). To test the hypotheses, we have examined the nonreproductive tissues in control and genomic ARKO males and females to: 1) determine the differences between males and females; 2) identify the genomic (DNA-binding-dependent) AR-mediated actions in males; 3 ) determine the contribution of genomic AR-mediated actions to these gender differences; and 4) identify physiological genomic AR-mediated actions in females.

\section{Materials and Methods}

\section{Mice}

ARKO males and females were studied on a C57BL/6 background, with all lines of mice used in this study backcrossed to a C57BL/6 background for $>8$ generations prior to experimental analysis. CMV-cre transgenic (CMVcre. $\mathrm{Tg}+$ ) mice were obtained with permission from Dr Ursula Lichtenberg, Institute for Genetics, University of Cologne. The $A R$ gene is on the $\mathrm{X}$ chromosome, and genomic ARKOs have exon 3 of the $A R$ gene deleted (AR ${ }^{\Delta}$; Notini et al. 2005). ARKO males $\left(\mathrm{AR}^{\Delta / \mathrm{Y}}\right.$ ) were offspring of heterozygous $\mathrm{AR}^{\Delta /+}$ females crossed with wild-type (WT) $\mathrm{AR}^{+/ \mathrm{Y}}$ males, and ARKO females were generated by crossing $\mathrm{AR}^{\Delta /+}$ and $\mathrm{CMV}$-cre. $\mathrm{Tg}+$ heterozygous females with $A R$ floxed $\left(A^{A / Y}\right)$ hemizygous males as described, which deletes $>99.9 \%$ of the AR in genomic DNA from ARKO females (MacLean et al. 2008). Control littermates were used for both males (WT) and females $\left(\mathrm{AR}^{\mathrm{fl}+}\right.$ and $\mathrm{CMV}$-cre. $\operatorname{Tg}+)$. Mice were housed in a conventional facility with a $12 \mathrm{~h}$ light: $12 \mathrm{~h}$ darkness cycle, and standard chow and water were provided ad libitum. Studies were conducted in accord with accepted standards of humane animal care, and performed with the approval of the Austin Health Animal Ethics Committee.

\section{Tissue collection and serum analysis}

Adult mice were studied at 9 weeks of age. For dynamic bone histomorphometry experiments, mice received two i.p. injections of $20 \mathrm{mg} / \mathrm{kg}$ per BW calcein (Sigma) at 10 and 3 days prior to killing. On the day of tissue harvest, mice were anesthetized by isoflurane and blood collected via cardiac puncture, and mice were killed by cervical dislocation. Blood was clotted on ice, and centrifuged $2 \times 1400 \mathrm{~g}$ to collect serum, which was stored at $-20{ }^{\circ} \mathrm{C}$ prior to analysis. Tissues were dissected post-mortem, and wet weight of tissues was determined to an accuracy of $0 \cdot 1 \mathrm{mg}$ from the mean mass bilaterally. Body mass and organ mass were measured in genomic ARKO and WT male littermates ( $n \geq 24$ /group), and in genomic ARKO females and $\mathrm{AR}^{\mathrm{f} /+}$ and CMVcre. $\mathrm{Tg}+$ female littermates ( $n \geq 12$ /group). Data from $\mathrm{AR}^{\mathrm{fl} /+}$ and $\mathrm{CMV}-\mathrm{cre} . \mathrm{Tg}+$ female controls were pooled, as there was no statistical difference between all parameters in these groups (data not shown). Since body weight was decreased in ARKOs and as the weights of most individual organs scale as a constant fraction of body mass (Lindstedt \& Schaeffer 2002), organ mass was analyzed as the absolute mass, and also relative mass, calculated by correcting the mass of individual organs by body weight. The C-terminal telopeptide $\alpha 1$ chain of type I collagen (X-laps) was determined in serum by ELISA (RatLaps ELISA; Nordic Bioscience Diagnostics, Herlev, Denmark). 


\section{Bone histomorphometry}

Femur length was measured using a digital calliper (Etalon, Renens, Switzerland). Distal femora were prepared for quantitative histomorphometry, using established resinembedding techniques as described previously (Notini et al. 2007). Trabecular bone volume, thickness, and number were calculated in the metaphyseal region below the growth plate excluding the primary spongiosa, using a Leica Quantimet Image Analysis System and QWin software (Cambridge, UK). The region of the metaphysis analyzed was a function of femur length as described previously (Davey et al. 2006, Notini et al. 2007). Dynamic markers of bone turnover (mineralizing surface, mineral apposition rate, bone formation rate, osteoclast surface, and osteoid surface) were estimated in the same region of the secondary spongiosa of the distal femoral metaphysis used for the static histomorphometry parameters as described (Davey et al. 2006, Notini et al. 2007).

\section{Quantitative micro-computed tomography}

The midshaft of the femur was evaluated using a microtomographic imaging system (Viva CT40; Scanco Medical AG, Bassersdorf, Switzerland) as described previously (Chiang et al. 2009). Fifty transverse computed tomography slices of the mid-femoral diaphysis were acquired using $10.5 \mu \mathrm{m}$ isotropic voxel size. Cortical bone properties were evaluated in a region commencing $56 \%$ of the femur length distal to the femoral head and extending $0.5 \mathrm{~mm}$ distally. Images were identified automatically, filtered, and reconstructed with a specimen-specific threshold of 240. Morphometric parameters were computed using a direct 2D and 3D approach that does not rely on any assumptions about the underlying structure (Hildebrand et al. 1999). Cortical thickness, periosteal circumference, and medullary circumference were determined.

\section{Statistical analysis}

When comparing the mean of two groups, two-tailed unpaired Student's $t$-test was used for equal or unequal variance where appropriate. A value of $P<0.05$ was considered significant. All analyses were performed using SPSS 16 for Mac (Chicago, IL, USA).

\section{Results}

\section{Control males versus control females}

To identify sexually dimorphic characteristics, we compared the mass of a number of potentially androgen-sensitive tissues in control male and female mice at 9 weeks of age. Males weighed 23\% $(P<0 \cdot 001)$ more than females (Fig. 1A), and absolute heart mass and kidney mass were higher in males than females $(19 \%$ higher, $P<0.001$ and $25 \%$ higher,
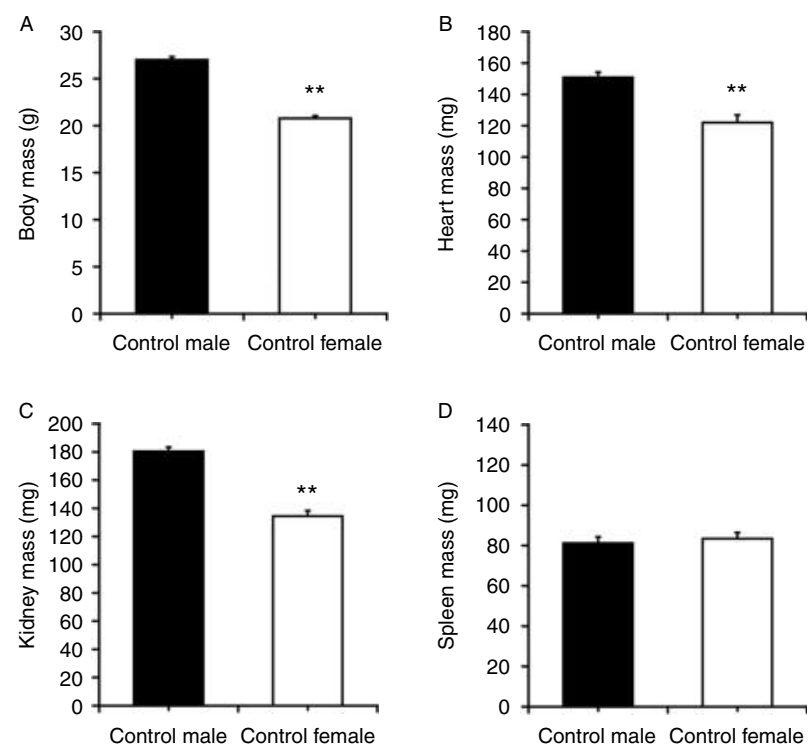

Figure 1 Body and organ mass in control males and females at 9 weeks of age. (A) Body mass, (B) absolute heart mass, (C) absolute kidney mass, and (D) absolute spleen mass. $n \geq 15$ /group,

$* * P<0 \cdot 001$ versus control male, Student's $t$-test.

$P<0 \cdot 001$ respectively; Fig. $1 \mathrm{~B}$ and $\mathrm{C})$. Heart mass and kidney mass were proportional to body mass, as there was no difference in heart weight/body weight ratio or kidney weight/body weight ratio between males and females (data not shown). Absolute spleen mass was not significantly different between males and females (Fig. 1D); however, relative spleen mass was $36 \%$ lower in males than females (mean \pm s.E.M.; control male: $2 \cdot 99 \pm 0.09 \mathrm{mg} / \mathrm{g}, n=22$ versus control female: $4 \cdot 08 \pm 0.12 \mathrm{mg} / \mathrm{g}, \quad n=25, \quad P<0 \cdot 001)$. Control males had increased femur length $(P<0 \cdot 001)$, periosteal circumference $(P<0 \cdot 005)$, cortical thickness $(P<0 \cdot 05)$, medullary circumference $(P<0 \cdot 05)$, trabecular bone volume $(P=0 \cdot 05)$, and trabecular number $(P<0 \cdot 001)$ compared with control females (Fig. 2).

\section{Genomic ARKO males versus control males}

To establish the actions of androgens mediated via the genomic AR signaling pathway in males, we compared ARKO males with their WT male littermate controls. Body mass was decreased by $16 \%(P<0 \cdot 001)$ in ARKO males compared to control males (Fig. 3A). Heart, kidney, and testis mass were reduced by $17 \%(P<0 \cdot 001), 28 \%(P<0 \cdot 001)$, and $94 \%(P<0 \cdot 001)$ respectively in ARKO males versus control males (Fig. 3B, C and E), whereas spleen mass was increased by $35 \%(P<0 \cdot 001$; Fig. 3D). In ARKO males, the decrease in heart mass was proportional to the decreased body mass (heart weight/body weight, mean \pm s.E.M.; control male: $5 \cdot 59 \pm 0.14 \mathrm{mg} / \mathrm{g}, n=30$ versus ARKO male: $5 \cdot 56$ $\pm 0 \cdot 12 \mathrm{mg} / \mathrm{g}, n=29)$, but relative kidney, spleen, and testis 

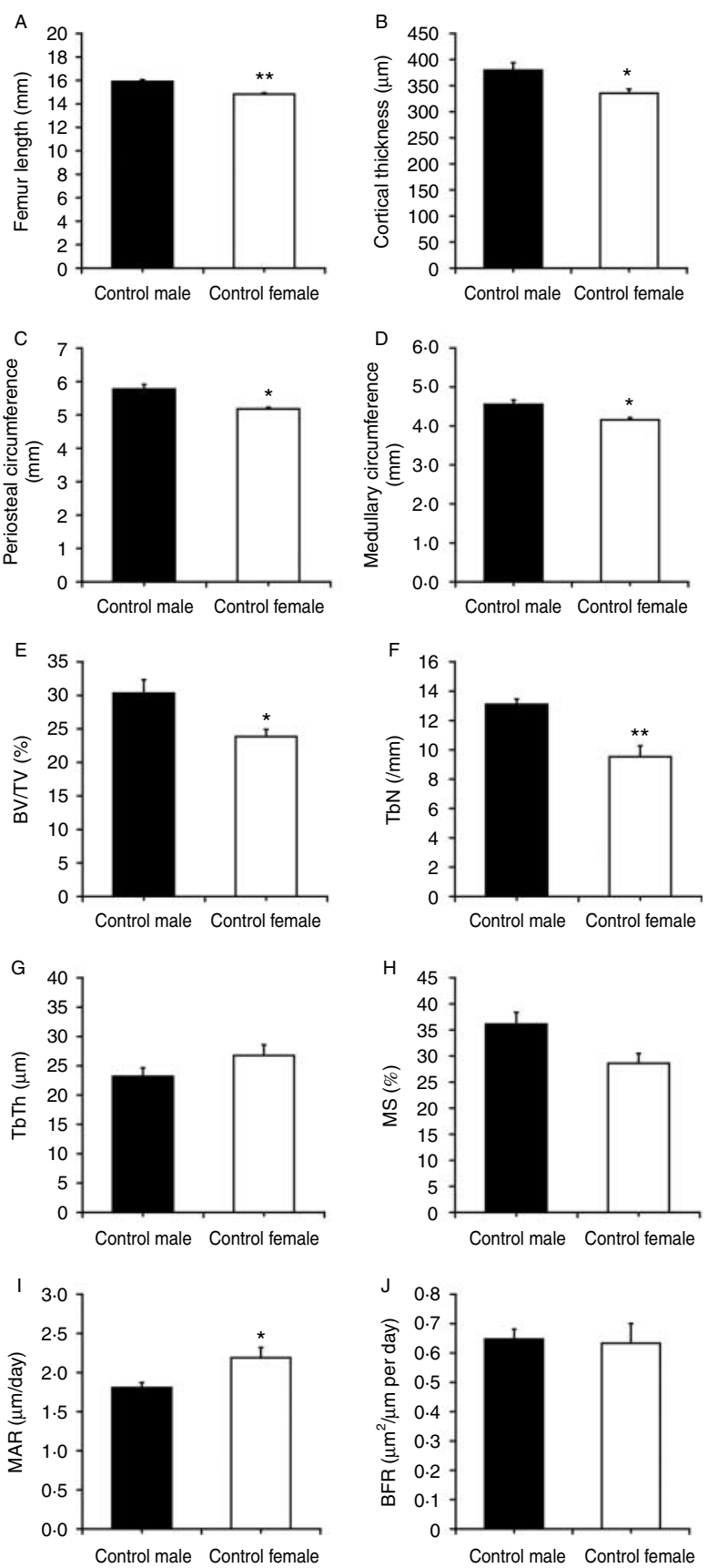

Figure 2 Bone parameters in control males and females at 9 weeks of age. (A) Femur length, (B) cortical thickness, (C) periosteal circumference, (D) medullary circumference. (E) trabecular bone volume (BV/TV), (F) trabecular number $(\mathrm{TbN}),(\mathrm{G})$ trabecular thickness (TbTh), (H) mineralizing surface (MS), (I) mineral apposition rate (MAR), and (J) bone formation rate (BFR). $n \geq 6$ /group, $* * P<0 \cdot 001, * P<0 \cdot 05$ versus control male, Student's $t$-test.

mass were all significantly different to control males (mean 土s.E.M.; kidney, control male: $6.64 \pm 0.08 \mathrm{mg} / \mathrm{g}, \quad n=36$ versus ARKO male: $5 \cdot 74 \pm 0.09 \mathrm{mg} / \mathrm{g}, n=36, P<0 \cdot 001$; spleen, control male: $2 \cdot 99 \pm 0.09 \mathrm{mg} / \mathrm{g}, n=22$ versus ARKO male: $4 \cdot 81 \pm 0 \cdot 15 \mathrm{mg} / \mathrm{g}, \quad n=26, \quad P<0 \cdot 001$; testis, control male: $3 \cdot 25 \pm 0 \cdot 08 \mathrm{mg} / \mathrm{g}, n=18$ versus ARKO male: $0 \cdot 22$ $\pm 0 \cdot 01 \mathrm{mg} / \mathrm{g}, n=21, P<0 \cdot 001)$.

ARKO males had decreases in both cortical and trabecular bone compared to control males, while femoral length was unchanged (Fig. 4A). Cortical thickness $(P<0 \cdot 005)$, periosteal circumference $(P<0 \cdot 005)$, and medullary circumference $(P<0 \cdot 05)$ were decreased in ARKO males by 14,11 , and $9 \%$ compared to control males respectively (Fig. 4B-D). Trabecular bone volume was also decreased by $33 \%$ in ARKO males $(P<0.001)$ compared to control males (Fig. 4E), due to a reduction in trabecular number $(P<0 \cdot 01$; Fig. 4F), not thickness (Fig. 4G). The reduced trabecular bone in ARKO males was associated with an increase in unmineralized matrix as measured by osteoid surface (mean \pm S.E.M.; control male: $10 \cdot 9 \pm 2 \cdot 7 \%, n=5$ versus ARKO male: $23.9 \pm 5 \cdot 4 \%, n=6 ; P<0 \cdot 05)$, and a reduction in mineralizing surface $(P<0 \cdot 01$; Fig. $4 \mathrm{H})$. Mineral apposition rate (MAR) was unaffected (Fig. 4I), and while the mean values for bone formation rate were lower in ARKO
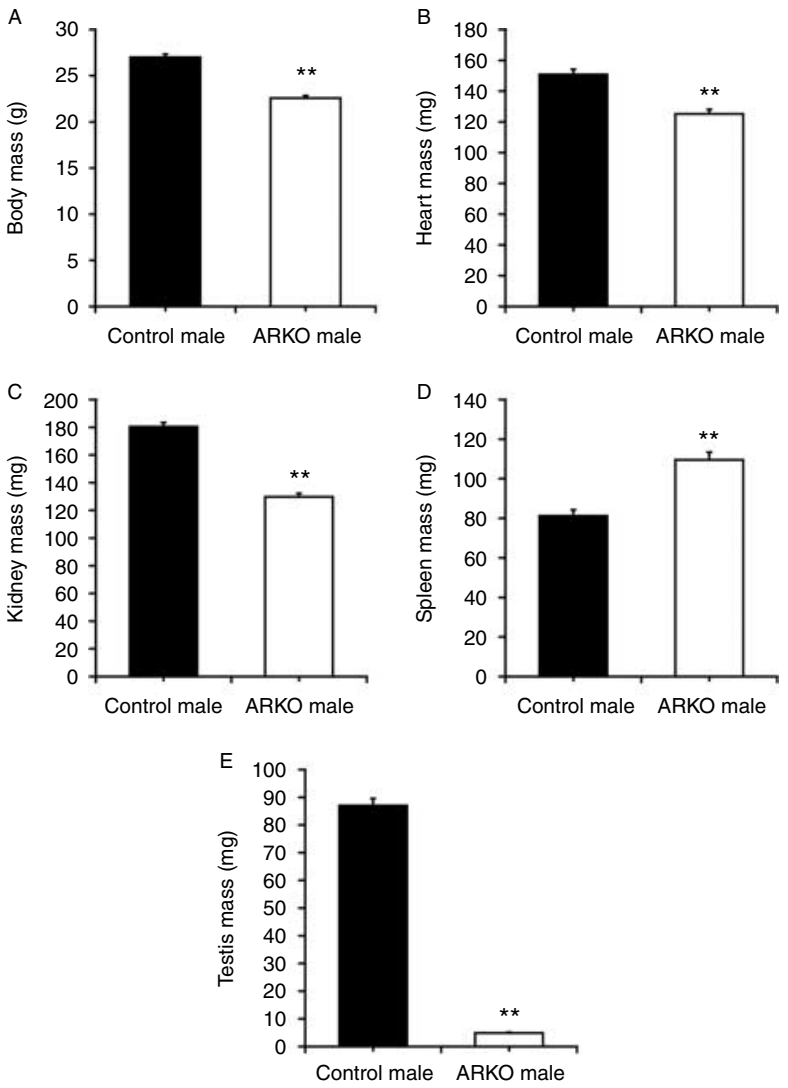

Figure 3 Body, organ, and reproductive tissue mass in ARKO males and control males at 9 weeks of age. (A) Body mass, (B) absolute heart mass, (C) absolute kidney mass, (D) absolute spleen mass, and (E) absolute testis mass. $n \geq 15 /$ group, $* * P<0 \cdot 001$ versus control male, Student's $t$-test. 

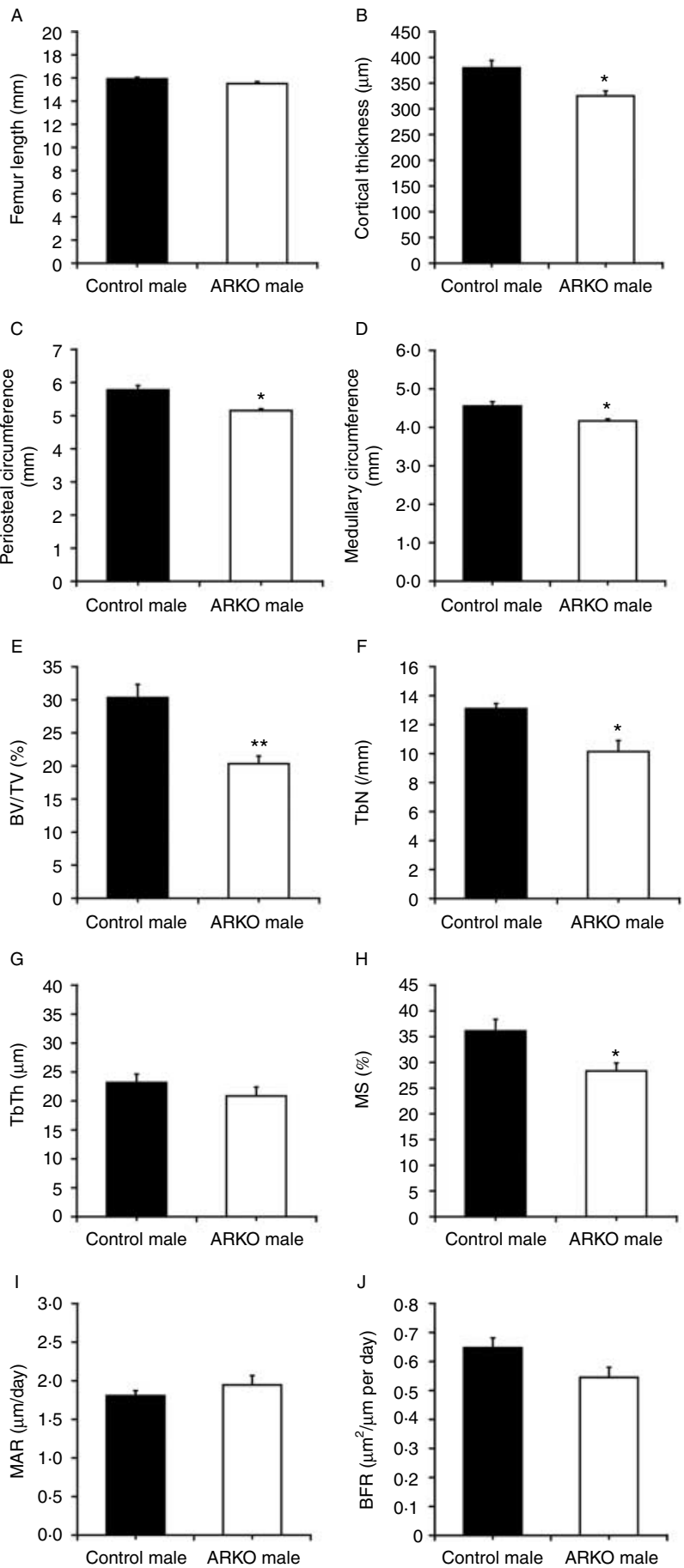

$\mathrm{H}$
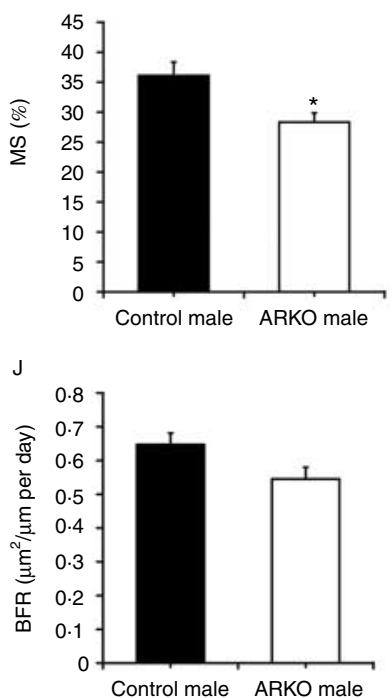

Figure 4 Bone parameters in ARKO males and control males at 9 weeks of age. (A) Femur length, (B) cortical thickness,

(C) periosteal circumference, (D) medullary circumference.

(E) trabecular bone volume (BV/TV), (F) trabecular number $(\mathrm{TbN})$,

(G) trabecular thickness (TbTh), $(\mathrm{H})$ mineralizing surface (MS),

(I) mineral apposition rate (MAR), and (J) bone formation rate

(BFR). $n \geq 6$ /group, ${ }^{* *} P<0 \cdot 001,{ }^{*} P<0 \cdot 05$ versus control male, Student's $t$-test. males compared to controls, this did not reach statistical significance $(P=0 \cdot 058$; Fig. $4 \mathrm{~J})$. Bone resorption as measured by osteoclast surface (mean \pm S.E.M.; control male: $7 \cdot 3 \pm 2 \cdot 1 \%$, $n=6$ versus ARKO male: $11 \cdot 9 \pm 3 \cdot 8 \%, n=5)$ and serum X-laps (mean \pm s.E.M.; control male: $32 \cdot 7 \pm 3 \cdot 5 \mathrm{ng} / \mathrm{ml}, n=14$ versus ARKO male: $41 \cdot 2 \pm 5 \cdot 1 \mathrm{ng} / \mathrm{ml}, n=14)$ were unchanged in ARKO males compared with controls.

\section{Genomic ARKO males versus control females}

To determine the contribution of the androgen/genomic AR pathway to the differences between males and females, we compared ARKO males with control females. Despite the decrease in body mass of ARKO males compared to control males, they were still $8 \%$ heavier than control females (Table 1). Both absolute heart mass and heart weight/body weight ratio did not differ between ARKO males and control females (data not shown). In contrast, while absolute kidney mass did not differ between ARKO males and control females, kidney weight/body weight ratio was $14 \%$ lower in ARKO males (Table 1), and both absolute and relative spleen mass were higher in ARKO males (Table 1). Femur length was increased in ARKO males (mean \pm s.E.M.; control females: $14 \cdot 8 \pm 0 \cdot 1, n=15$ versus ARKO males: $15 \cdot 5 \pm 0 \cdot 2 \mathrm{~mm}, n=9$ versus $P<0 \cdot 001)$, while trabecular bone volume was decreased (mean \pm s.E.M.; control females: $30 \cdot 3 \pm 2 \cdot 0, n=8$ versus ARKO males: $20 \cdot 3 \pm 1 \cdot 2, n=10$, $P<0 \cdot 05)$, due to decreased trabecular thickness in ARKO males (mean \pm s.E.M.; control females: $21 \pm 2 \mu \mathrm{m}, n=9$ versus ARKO males: $23 \pm 1 \mu \mathrm{m}, n=8, P<0 \cdot 05)$. Cortical bone and the dynamic bone parameters did not differ between ARKO males and control females (data not shown).

\section{Genomic ARKO females versus control females}

To identify physiological actions of androgens in females, we compared ARKO females with control females. There was no difference in the body mass of ARKO females and control females (Fig. 5A). Despite the fact that total body mass did not differ, absolute heart mass was decreased by $14 \%$ in ARKO females (mean \pm s.E.M.; control female: $122.0 \pm 4.9 \mathrm{mg}$,

Table 1 Comparison of 9-week-old genomic ARKO male mice with control female mice. Data represent mean \pm s.E.M.
Body mass (g)

Kidney weight/body weight ratio $(\mathrm{mg} / \mathrm{g})$

Spleen weight $(\mathrm{g})$

Spleen weight/body

weight ratio $(\mathrm{mg} / \mathrm{g})$

\section{Control female}

$20 \cdot 8 \pm 0 \cdot 3(37)^{\mathrm{a}}$

$6 \cdot 56 \pm 0 \cdot 12(26)$

$83 \cdot 4 \pm 3 \cdot 1(25)$

$4 \cdot 08 \pm 0 \cdot 12(25)$
ARKO male

$22 \cdot 6 \pm 0 \cdot 3 * *(53)$

$5 \cdot 74 \pm 0 \cdot 09^{* *}(36)$

$109 \cdot 6 \pm 3 \cdot 9^{* *}(26)$

$4 \cdot 81 \pm 0 \cdot 15^{* *}(26)$
ARKO, androgen receptor knockout.

${ }^{\mathrm{a}}$ Number in parenthesis denotes sample size.

${ }^{* *} P<0 \cdot 001$ versus control females, Student's $t$-test. 

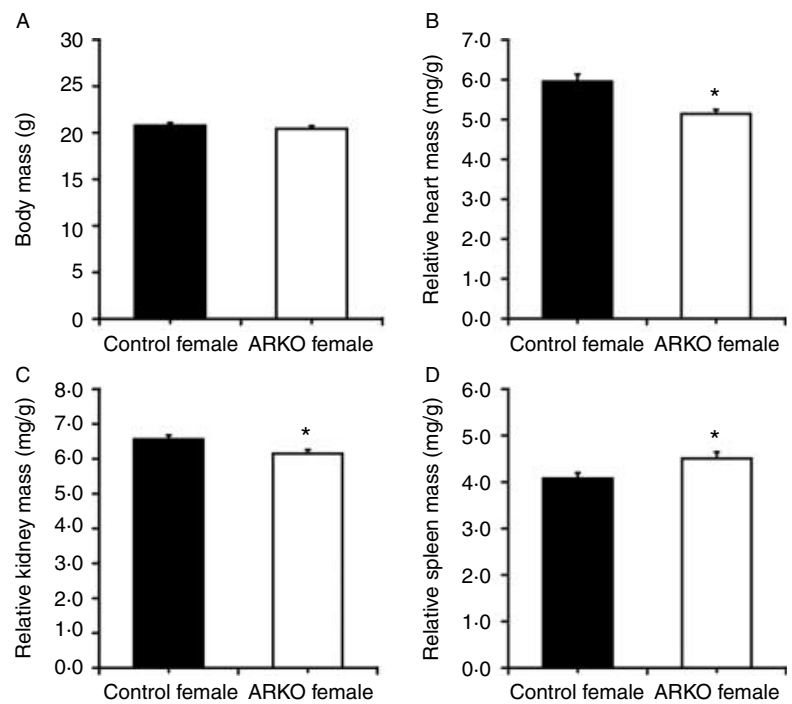

Figure 5 Body and organ mass in ARKO females and control females at 9 weeks of age. (A) Body mass, (B) relative heart mass, (C) relative kidney mass, and (D) relative spleen mass. $n \geq 15 /$ group, $* P<0.05$ versus control female, Student's $t$-test.

$n=25$ versus ARKO female: $104 \cdot 9 \pm 2 \cdot 2 \mathrm{mg}, n=12$, $P=0 \cdot 001)$, and heart weight/body weight ratio was also reduced by the same amount $(P<0 \cdot 01$; Fig. $5 \mathrm{~B})$. Relative kidney mass was decreased by $6 \%(P<0 \cdot 05)$ in $\mathrm{ARKO}$ females (Fig. 5C), with a trend for reduced absolute kidney mass (mean \pm s.E.M.; control female: $134 \cdot 5 \pm 3 \cdot 8 \mathrm{mg}, n=26$ versus ARKO female: $125 \cdot 8 \pm 3 \cdot 6 \mathrm{mg}, n=12, P=0 \cdot 082)$. Similarly, relative spleen mass was increased by $10 \cdot 5 \%$ $(P<0 \cdot 05)$ in ARKO females (Fig. 5D), with a trend for increased absolute spleen mass (mean \pm s.E.M.; control female: $83.4 \pm 3.1 \mathrm{mg}, n=25$ versus ARKO female: $92.4 \pm 3.9 \mathrm{mg}$, $n=12, P=0 \cdot 095)$. There was no difference between ARKO females and control females in absolute or relative ovary or uterus mass (data not shown).

Periosteal circumference and medullary circumference were decreased by 4 and 5\% respectively in ARKO females compared to controls $(P<0 \cdot 05)$, while cortical thickness was unaffected (Fig. 6B-D). Although trabecular bone volume was unaffected in ARKO females compared to controls (Fig. 6E), trabecular number was decreased by $26 \%(P<0 \cdot 01$; Fig. 6F), and trabecular thickness was increased by $15 \%$ $(P<0.05)$ (Fig. 6G). No differences were observed in measurements of bone formation (mineralizing surface, mineral apposition rate, and bone formation rate; Fig. $6 \mathrm{H}-\mathrm{J}$ ).

\section{Discussion}

To determine the contribution of androgens acting through the genomic (DNA-binding-dependent) AR signaling pathway in males and females to gender differences, we generated and characterized male and female mice with ablation of the genomic actions of the AR. Other global ARKO mouse models are completely AR null, because they have frameshift deletions that result in no detectable AR
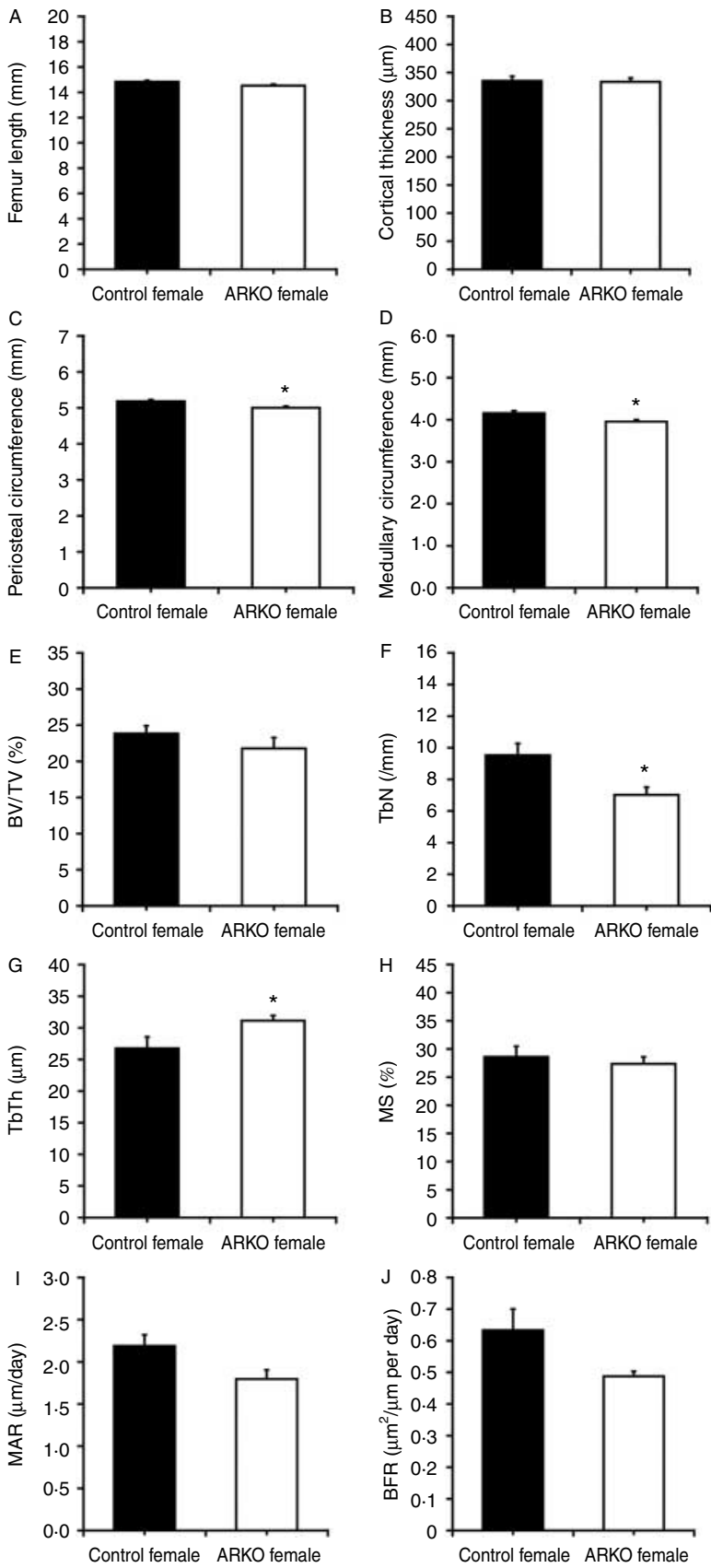

Figure 6 Bone parameters in ARKO females and control females at 9 weeks of age. (A) Femur length (B) cortical thickness,

(C) periosteal circumference, (D) medullary circumference,

(E) trabecular bone volume (BV/TV), $(\mathrm{F})$ trabecular number $(\mathrm{TbN})$, (G) trabecular thickness (TbTh), (H) mineralizing surface (MS), (I) mineral apposition rate (MAR), and (J) bone formation rate (BFR). $n \geq 6$ /group, $* P<0 \cdot 05$ versus control female, Student's $t$-test. 
protein, and therefore have deletion of both genomic (DNA-binding-dependent) and DNA-binding-independent AR signaling pathways (Yeh et al. 2002, Kawano et al. 2003, De Gendt et al. 2004). In contrast, our ARKO mice express mutant AR protein, lacking the second zinc finger of the DNA-binding domain (Notini et al. 2005). We previously showed that our genomic ARKO mice have a modest reduction in serum testosterone levels (Notini et al. 2005); therefore, their phenotype arises through a combination of loss of genomic AR signaling and testosterone deficiency. However, treatment of genomic ARKO males with supraphysiological levels of testosterone for 6 weeks has no effect on any phenotypic parameter examined ((Lim et al. 2008), and H E MacLean \& R A Davey, unpublished data), supporting the hypothesis that the ARKO male phenotype is caused by the loss of DNA-binding-dependent AR actions. Therefore, our study is strongly suggestive that androgens act through the genomic AR signaling pathway to play a physiological role in a number of tissues in both sexes.

Body mass of ARKO males was between that of normal males and females. We previously showed that ARKO males have normal levels of serum insulin-like growth factor 1 (MacLean et al. 2008), and the fact that femoral length did not differ between ARKO and control males suggests that skeletal growth rate is normal. We have also previously demonstrated that both absolute and relative skeletal muscle mass are decreased in ARKO males versus control males (MacLean et al. 2008). Since body mass is contributed by lean body mass (muscle and visceral organs), fat mass, and skeletal size and density, the reduction in ARKO male body mass is therefore most likely to be caused by the reduction in mass of skeletal muscle and other organs. Body weight and femoral length were still higher in ARKO males than control females, in contrast to previous studies showing no difference between young AR-null ARKO males and control females (Sato et al. 2003, Lin et al. 2005). A major difference between AR-null ARKO models and our genomic ARKO mice is that the genomic ARKOs retain potential DNAbinding-independent AR actions. These include activation of second-messenger signaling pathways by the AR (Heinlein \& Chang 2002), and indirect gene repression in the absence of direct binding to DNA, via tethering of transcription factors (Schneikert et al. 1996). While our study was not designed to identify AR actions independent of DNA binding, the differences in ARKO male body mass between the different models suggest the possibility that this could contribute to promoting body growth in males. However, to definitively identify the role of non-DNA-binding-dependent AR actions, direct comparisons between the different ARKO models would be required to be performed in the same environment, and with the mice on the same genetic background. The fact that genomic ARKO males were larger than females could also be analogous to the height of patients with complete AIS, which has been reported as increased compared to average female height (Marcus et al. 2000). Alternatively, the increased longitudinal growth in ARKO males compared with control females may be attributed to the lower levels of circulating testosterone in the ARKO males that is available for conversion to estradiol (Yamazaki \& Yamaguchi 1989, Notini et al. 2005). Further supportive evidence for this hypothesis is the lower trabecular bone volume and thickness in the ARKO males compared with control females, which are changes consistent with estrogen deficiency in the rodent (Wronski et al. 1986, Yamazaki \& Yamaguchi 1989). However, in combination with these previous studies, our data suggest that factors other than the genomic AR pathway contribute to the size and height differential between males and females.

Cardiac mass is sexually dimorphic, with our data showing that males had larger hearts than females, in proportion to their differences in body weight. Androgens promote cardiac myocyte hypertrophy in vitro (Marsh et al. 1998), and male mice that are AR null have small hearts, with a $14 \%$ decrease in heart weight/body weight ratio, and are susceptible to angiotensin II-induced cardiac remodeling (Ikeda et al. 2005). In contrast, our ARKO males had a $17 \%$ decrease in absolute cardiac mass compared with control males, but heart weight/ body weight ratio was unchanged. The milder cardiac phenotype in our ARKO males compared with AR-null ARKO males may reflect the effects of different genetic backgrounds (homogenous C57BL/6 in our study versus mixed C57BL/6 and CBA in the previous study). Alternatively, these differences could also indicate the possibility that a DNA-binding-independent AR signaling pathway acts in concert with the classical genomic pathway in the heart to promote cardiac growth and development. This latter hypothesis is supported by the fact that rapid androgen signaling pathways have been identified in cardiomyocytes in vitro (Vicencio et al. 2006), and a recent study has showed that DHT treatment of isolated rat left atria causes a non-transcriptional cardiotonic response associated with increased ornithine decarboxylase (ODC) activity (Bordallo et al. 2009). The fact that there was no difference in absolute or relative heart mass between ARKO males and control females indicates that the sex differences in cardiac mass are determined solely by androgens acting through the genomic AR pathway in males.

In mice, the kidney is one of the most androgen-sensitive non-reproductive tissues, with kidney mass closely related to androgen levels in males (Berger \& Watson 1989). Kidney mass was $25 \%$ lower in female mice than males, proportional to the lower body mass in females. This may reflect the fact that lower body mass in females is caused by the decrease in both visceral organ and skeletal muscle mass compared to males. ARKO males also had a reduction in both absolute and relative kidney mass compared to control males, and ARKO male kidney mass was reduced to that of normal females, supporting the hypothesis that the gender differences in kidney mass are due to androgen actions in males. Androgens are thought to regulate kidney hypertrophy in part through AR-dependent regulation of polyamine levels, demonstrated by the fact that androgens regulate the genes 
encoding polyamine biosynthetic enzymes including ODC in the kidney (Levillain et al. 2005). Androgen response elements have also been identified in the $O d c$ gene promoter (Crozat et al. 1992). Together, these data suggest that androgens promote kidney hypertrophy in males through the genomic AR pathway.

Females have a higher incidence of autoimmune diseases than males, and sex hormones regulate the immune system (McCombe et al. 2009). Castration of male mice increases splenic and thymic weights, due to an increase in newly formed B cells and mature B lymphocytes (Ellis et al. 2001). Male Tfm mice, with the naturally occurring AR mutation, also have increased $\mathrm{B}$ cell precursors in the bone marrow and mature B cells in the spleen (Smithson et al. 1998), and our data demonstrating increased splenic weight in genomic ARKO males compared to control males suggest that the suppression of B lymphopoiesis by androgens occurs via the genomic AR pathway. ARKO males also had higher absolute and relative splenic weights than control females. Female hypogonadal (hpg) mice, which lack GnRH and therefore have a deficiency in both estrogen and androgen production, have elevated B lymphopoiesis and increased splenic B cell number, which can be normalized by estrogen supplementation (Smithson et al. 1994). Therefore, the differences in splenic weight between males and females are likely to be due to androgen actions in males and also estrogen-mediated suppression of lymphopoiesis in females.

Males have larger bones than females, in part due to the anabolic actions of androgens on outward bone growth via stimulation of periosteal apposition (Seeman 2001). In females, estrogens inhibit this process while stimulating endocortical deposition of bone (Seeman 2001). Consistent with this, cortical thickness, periosteal circumference, and medullary circumference were decreased in ARKO males compared to control males. Furthermore, the loss of genomic AR action in ARKO males resulted in a 33\% reduction in trabecular bone volume compared to controls, and was due to fewer trabeculae, while trabecular thickness was unaffected. A similar reduction in trabecular bone volume has been observed in AR-null males, due to increased bone resorption (Yeh et al. 2002, Kawano et al. 2003, Venken et al. 2006). Despite the decreased trabecular bone volume in our ARKO males, we did not detect any increase in bone resorption markers. It is possible that bone resorption is also increased in our ARKO males prior to 8 weeks of age, as suggested by our osteoblast-specific ARKOs (Notini et al. 2007, Chiang et al. 2009). The decreased trabecular bone in the ARKO males was also attributed to an increase in unmineralized bone matrix and a decrease in the number of mineralizing surfaces reflected by osteoid surface and mineralizing surface respectively, providing evidence for a role of androgens via the AR in the coordination of bone matrix synthesis with its subsequent mineralization. The trabecular bone phenotype of the ARKO males is consistent with our previous findings that deletion of genomic AR signaling specifically in mature and mineralizing osteoblasts results in trabecular bone loss with increased bone resorption (Notini et al. 2007, Chiang et al. 2009), and that deletion in mineralizing osteoblasts results in disruption of the bone matrix synthesis and mineralization processes (Chiang et al. 2009). Taken together, these data indicate the importance of genomic AR signaling in males to maintain cortical and trabecular bones.

The physiological actions of androgens via the genomic AR signaling pathway in females have not previously been well understood, due to the lack of good knockout mouse models, and the inability to distinguish androgen and estrogen effects in gonadectomy studies. Although females have low levels of circulating androgens, the local androgen concentrations in tissues can be higher, and most tissues express the AR at levels similar to that found in males (Rinn et al. 2004), providing potential for significant androgen actions in females. The use of the cre/lox approach has enabled the generation of global ARKO females to identify the physiological actions of androgens. Previous studies in ARKO females have focused only on reproductive actions, including identifying a role for androgens in folliculogenesis, mammary gland development, and uterine morphology and development (Yeh et al. 2003, Hu et al. 2004, Shiina et al. 2006, Walters et al. 2007, 2009). Despite the defect in late follicular development, our genomic ARKO female mice have normal levels of serum LH, FSH, estradiol, and testosterone (Walters et al. 2007). Our current study demonstrates that in addition to these reproductive actions, androgens also act through the genomic AR pathway in female kidney, heart, bone marrow, and bone.

In females, there was no difference in body mass between ARKOs and controls, despite the change in mass of some visceral organs, indicating that the effects of androgens on body mass are male specific. This is consistent with the findings in AR-null female models (Yeh et al. 2003, Shiina et al. 2006). ARKO females had a $14 \%$ decrease in absolute cardiac mass and heart weight/body weight ratio compared to control females. Therefore, androgens also play a physiological role in promoting cardiac growth in females. Relative kidney mass was reduced by $6 \%$ in ARKO females compared to control females. While this reduction is only half of that observed in ARKO males compared to control males, it nevertheless demonstrates that androgens also promote kidney hypertrophy in normal females. ARKO females also had increased relative splenic mass compared to control females. Therefore, this suggests that androgens acting via the genomic AR pathway play a physiological role to downregulate $\mathrm{B}$ lymphopoiesis in females.

Femoral length was unaffected in our ARKO males and females, consistent with observations in AR-null mouse models (Kawano et al. 2003, Venken et al. 2006). Of interest was the small, but significant decrease in bone size of ARKO females, with decreases in periosteal circumference and medullary circumference. This is the first report of a possible role of the AR in cortical bone growth in female mice, as previous characterization of the bone phenotype of AR-null ARKO female mice has been limited to soft X-ray and bone 
mineral density analyses (Kawano et al. 2003). Androgen actions result in the outward growth of bone via stimulation of periosteal apposition in males, while estrogens inhibit this process and stimulate endocortical deposition in females (Seeman 2001, Jarvinen et al. 2003). Although the actions of estrogen are predominant in females, these data also provide evidence for a contribution of androgen action via the genomic AR pathway in cortical bone growth and expansion in females.

While trabecular bone volume of ARKO females did not differ from control females, we observed a reduction in trabecular number and increase in trabecular thickness, suggestive of increased bone turnover. Therefore, while estrogen is the main sex steroid regulating bone accrual and maintenance in females, we have provided evidence that androgens also play a physiological role in bone in females via the genomic AR pathway. Supportive evidence for this is provided by observations of increased bone mineral density in hirsute women with increased circulating levels of testosterone (Buchanan et al. 1988), in woman following treatment with the synthetic non-aromatizable androgen nandrolone decanoate (Need et al. 1987, Johansen et al. 1989), and in female ovariectomized rats treated with the non-aromatizable androgen, DHT (Tobias et al. 1994).

In conclusion, we have identified that the genomic AR signaling pathway mediates, at least in part, the gender differences in body mass, heart, kidney, spleen, and bone. In addition, this study provides the first evidence that androgens acting via the genomic AR pathways in females play a physiological role in the regulation of splenic mass, cardiac growth, kidney hypertrophy, cortical bone growth, and trabecular bone architecture. We have shown that DNA-binding-dependent AR actions play a role in cortical and trabecular bone accrual and maintenance in females, and we have also identified that heart, kidney, and spleen are key areas of AR action outside the musculoskeletal system. In-depth analysis of these other organs will provide further information regarding the mechanisms via which genomic AR signaling regulates their function.

\section{Declaration of interest}

The authors declare that there is no conflict of interest that could be perceived as prejudicing the impartiality of the research reported.

\section{Funding}

This research was supported by NHMRC Project Grants \#350334 and \#350346 and an Eva and Les Erdi Research Grant; HEM was supported by NHMRC Career Development Award \#359226.

\section{Acknowledgements}

We thank Ursula Lichtenburg for the CMV-cre mice and Rebecca Sawyer of the Hanson Institute for her excellent technical assistance.

\section{References}

Axell AM, MacLean HE, Plant DR, Harcourt LJ, Davis JA, Jimenez M, Handelsman DJ, Lynch GS \& Zajac JD 2006 Continuous testosterone administration prevents skeletal muscle atrophy and enhances resistance to fatigue in orchidectomized male mice. American Journal of Physiology. Endocrinology and Metabolism 291 E506-E516.

Behre HM, Kliesch S, Leifke E, Link TM \& Nieschlag E 1997 Long-term effect of testosterone therapy on bone mineral density in hypogonadal men. Journal of Clinical Endocrinology and Metabolism 82 2386-2390.

Berger FG \& Watson G 1989 Androgen-regulated gene expression. Annual Review of Physiology 51 51-65.

Bordallo J, Secades L, Bordallo C, Cantabrana B \& Sanchez M 2009 Influence of gender and sex hormones on 5alpha-dihydrotestosterone elicited effect in isolated left atria of rats: role of beta-adrenoceptors and ornithine decarboxylase activity. European Journal of Pharmacology 604 103-110.

Buchanan JR, Hospodar P, Myers C, Leuenberger P \& Demers LM 1988 Effect of excess endogenous androgens on bone density in young women. Journal of Clinical Endocrinology and Metabolism 67 937-943.

Chen Y, Zajac JD \& MacLean HE 2005 Androgen regulation of satellite cell function. Journal of Endocrinology 186 21-31.

Chiang C, Chiu M, Moore AJ, Anderson PH, Ghasem-Zadeh A, McManus JF, Ma C, Seeman E, Clemens TL, Morris HA et al. 2009 Mineralization and bone resorption are regulated by the androgen receptor in male mice. Journal of Bone and Mineral Research 24 621-631.

Colvard DS, Eriksen EF, Keeting PE, Wilson EM, Lubahn DB, French FS, Riggs BL \& Spelsberg TC 1989 Identification of androgen receptors in normal human osteoblast-like cells. PNAS 86 854-857.

Crozat A, Palvimo JJ, Julkunen M \& Jänne OA 1992 Comparison of androgen regulation of ornithine decarboxylase and $S$-adenosylmethionine decarboxylase gene expression in rodent kidney and accessory sex organs. Endocrinology 130 1131-1144.

Davey RA, Moore AJ, Chiu MW, Notini AJ, Morris HA \& Zajac JD 2006 Effects of amylin deficiency on trabecular bone in young mice are sex-dependent. Calcified Tissue International 78 398-403.

De Gendt K, Swinnen JV, Saunders PT, Schoonjans L, Dewerchin M, Devos A, Tan K, Atanassova N, Claessens F, Lecureuil C et al. 2004 A Sertoli cell-selective knockout of the androgen receptor causes spermatogenic arrest in meiosis. PNAS 101 1327-1332.

Ellis TM, Moser MT, Le PT, Flanigan RC \& Kwon ED 2001 Alterations in peripheral B cells and B cell progenitors following androgen ablation in mice. International Immunology 13 553-558.

Estrada M, Espinosa A, Muller M \& Jaimovich E 2003 Testosterone stimulates intracellular calcium release and mitogen-activated protein kinases via a $G$ protein-coupled receptor in skeletal muscle cells. Endocrinology 144 3586-3597.

Gill A, Jamnongit M \& Hammes SR 2004 Androgens promote maturation and signaling in mouse oocytes independent of transcription: a release of inhibition model for mammalian oocyte meiosis. Molecular Endocrinology 18 97-104.

Grumbach MM \& Auchus RJ 1999 Estrogen: consequences and implications of human mutations in synthesis and action. Journal of Clinical Endocrinology and Metabolism 84 4677-4694.

Handelsman DJ 2010 Androgen physiology, pharmacology and abuse. In Endocrinology, edn 6, pp 2469-2498. Eds DeGroot LJ \& Jameson JL. Philadelphia: Elsevier Saunders.

Heinlein CA \& Chang C 2002 The roles of androgen receptors and androgen-binding proteins in nongenomic androgen actions. Molecular Endocrinology 16 2181-2187.

Hildebrand T, Laib A, Muller R, Dequeker J \& Ruegsegger P 1999 Direct three-dimensional morphometric analysis of human cancellous bone: microstructural data from spine, femur, iliac crest, and calcaneus. Journal of Bone and Mineral Research 14 1167-1174.

Hu YC, Wang PH, Yeh S, Wang RS, Xie C, Xu Q, Zhou X, Chao HT, Tsai MY \& Chang C 2004 Subfertility and defective folliculogenesis in female mice lacking androgen receptor. PNAS 101 11209-11214. 
Ikeda Y, Aihara K, Sato T, Akaike M, Yoshizumi M, Suzaki Y, Izawa Y, Fujimura M, Hashizume S, Kato M et al. 2005 Androgen receptor gene knockout male mice exhibit impaired cardiac growth and exacerbation of angiotensin II-induced cardiac fibrosis. Journal of Biological Chemistry 280 29661-29666.

Jarvinen TL, Kannus P \& Sievanen H 2003 Estrogen and bone - a reproductive and locomotive perspective. Journal of Bone and Mineral Research 18 1921-1931.

Johansen JS, Hassager C, Podenphant J, Riis BJ, Hartwell D, Thomsen K \& Christiansen C 1989 Treatment of postmenopausal osteoporosis: is the anabolic steroid nandrolone decanoate a candidate? Bone and Mineral $677-86$.

Jones ME, Thorburn AW, Britt KL, Hewitt KN, Wreford NG, Proietto J, $\mathrm{Oz}$ OK, Leury BJ, Robertson KM, Yao S et al. 2000 Aromatase-deficient (ArKO) mice have a phenotype of increased adiposity. PNAS 97 12735-12740.

Kang HY, Cho CL, Huang KL, Wang JC, Hu YC, Lin HK, Chang C \& Huang KE 2004 Nongenomic androgen activation of phosphatidylinositol 3-kinase/Akt signaling pathway in MC3T3-E1 osteoblasts. Journal of Bone and Mineral Research 19 1181-1190.

Kawano H, Sato T, Yamada T, Matsumoto T, Sekine K, Watanabe T, Nakamura T, Fukuda T, Yoshimura K, Yoshizawa Tet al. 2003 Suppressive function of androgen receptor in bone resorption. PNAS 100 9416-9421.

Kousteni S, Bellido T, Plotkin LI, O'Brien CA, Bodenner DL, Han L, Han K, DiGregorio GB, Katzenellenbogen JA, Katzenellenbogen BS et al. 2001 Nongenotropic, sex-nonspecific signaling through the estrogen or androgen receptors: dissociation from transcriptional activity. Cell 104 719-730.

Levillain O, Diaz JJ, Blanchard O \& Dechaud H 2005 Testosterone down-regulates ornithine aminotransferase gene and up-regulates arginase II and ornithine decarboxylase genes for polyamines synthesis in the murine kidney. Endocrinology 146 950-959.

Lim P, Allan CM, Notini AJ, Axell AM, Spaliviero J, Jimenez M, Davey R, McManus J, MacLean HE, Zajac JD et al. 2008 Oestradiol-induced spermatogenesis requires a functional androgen receptor. Reproduction, Fertility, and Development 20 861-870.

Lin HY, Xu Q, Yeh S, Wang RS, Sparks JD \& Chang C 2005 Insulin and leptin resistance with hyperleptinemia in mice lacking androgen receptor. Diabetes 54 1717-1725.

Lindstedt SL \& Schaeffer PJ 2002 Use of allometry in predicting anatomical and physiological parameters of mammals. Laboratory Animals $361-19$.

MacLean HE, Warne GL \& Zajac JD 1997 Localization of functional domains in the androgen receptor. Journal of Steroid Biochemistry and Molecular Biology 62 233-242.

MacLean HE, Ball EM, Rekaris G, Warne GL \& Zajac JD 2004 Novel androgen receptor gene mutations in Australian patients with complete androgen insensitivity syndrome. Human Mutation 23287.

MacLean HE, Chiu WS, Notini AJ, Axell AM, Davey RA, McManus JF, Ma C, Plant DR, Lynch GS \& Zajac JD 2008 Impaired skeletal muscle development and function in male, but not female, genomic androgen receptor knockout mice. FASEB Journal 22 2676-2689.

Marcus R, Leary D, Schneider DL, Shane E, Favus M \& Quigley CA 2000 The contribution of testosterone to skeletal development and maintenance: lessons from the androgen insensitivity syndrome. Journal of Clinical Endocrinology and Metabolism 85 1032-1037.

Marsh JD, Lehmann MH, Ritchie RH, Gwathmey JK, Green GE \& Schiebinger RJ 1998 Androgen receptors mediate hypertrophy in cardiac myocytes. Circulation 98 256-261.

McCombe PA, Greer JM \& Mackay IR 2009 Sexual dimorphism in autoimmune disease. Current Molecular Medicine 9 1058-1079.

Mooradian AD, Morley JE \& Korenman SG 1987 Biological actions of androgens. Endocrine Reviews 8 1-28.

Need AG, Morris HA, Hartley TF, Horowitz M \& Nordin BE 1987 Effects of nandrolone decanoate on forearm mineral density and calcium metabolism in osteoporotic postmenopausal women. Calcified Tissue International $\mathbf{4 1} 7-10$
Nisenblat V \& Norman RJ 2009 Androgens and polycystic ovary syndrome. Current Opinion in Endocrinology, Diabetes and Obesity 16 224-231.

Norris JD, Chang CY, Wittmann BM, Kunder RS, Cui H, Fan D, Joseph JD \& McDonnell DP 2009 The homeodomain protein HOXB13 regulates the cellular response to androgens. Molecular Cell 36 405-416.

Notini AJ, Davey RA, McManus JF, Bate KL \& Zajac JD 2005 Genomic actions of the androgen receptor are required for normal male sexual differentiation in a mouse model. Journal of Molecular Endocrinology 35 547-555.

Notini AJ, McManus JF, Moore A, Bouxsein M, Jimenez M, Chiu WS, Glatt V, Kream BE, Handelsman DJ, Morris HA et al. 2007 Osteoblast deletion of exon 3 of the androgen receptor gene results in trabecular bone loss in adult male mice. Journal of Bone and Mineral Research 22 347-356.

Oz OK, Hirasawa G, Lawson J, Nanu L, Constantinescu A, Antich PP, Mason RP, Tsyganov E, Parkey RW, Zerwekh JE et al. 2001 Bone phenotype of the aromatase deficient mouse. Journal of Steroid Biochemistry and Molecular Biology 79 49-59.

Rinn JL, Rozowsky JS, Laurenzi IJ, Petersen PH, Zou K, Zhong W, Gerstein M \& Snyder M 2004 Major molecular differences between mammalian sexes are involved in drug metabolism and renal function. Developmental Cell 6 791-800.

Robertson KM, Simpson ER, Lacham-Kaplan O \& Jones ME 2001 Characterization of the fertility of male aromatase knockout mice. Journal of Andrology 22 825-830.

Sato T, Matsumoto T, Yamada T, Watanabe T, Kawano H \& Kato S 2003 Late onset of obesity in male androgen receptor-deficient (ARKO) mice. Biochemical and Biophysical Research Communications 300 167-171.

Schneikert J, Peterziel H, Defossez PA, Klocker H, Launoit Y \& Cato AC 1996 Androgen receptor-Ets protein interaction is a novel mechanism for steroid hormone-mediated down-modulation of matrix metalloproteinase expression. Journal of Biological Chemistry 271 23907-23913.

Seeman E 2001 Clinical review 137: sexual dimorphism in skeletal size, density, and strength. Journal of Clinical Endocrinology and Metabolism 86 4576-4584.

Shiina H, Matsumoto T, Sato T, Igarashi K, Miyamoto J, Takemasa S, Sakari M, Takada I, Nakamura T, Metzger D et al. 2006 Premature ovarian failure in androgen receptor-deficient mice. PNAS 103 224-229.

Smith EP, Boyd J, Frank GR, Takahashi H, Cohen RM, Specker B, Williams TC, Lubahn DB \& Korach KS 1994 Estrogen resistance caused by a mutation in the estrogen-receptor gene in a man. New England Journal of Medicine 331 1056-1061.

Smithson G, Beamer WG, Shultz KL, Christianson SW, Shultz LD \& Kincade PW 1994 Increased B lymphopoiesis in genetically sex steroid-deficient hypogonadal (hpg) mice. Journal of Experimental Medicine 180 717-720.

Smithson G, Couse JF, Lubahn DB, Korach KS \& Kincade PW 1998 The role of estrogen receptors and androgen receptors in sex steroid regulation of $\mathrm{B}$ lymphopoiesis. Journal of Immunology 161 27-34.

Strauss RH, Liggett MT \& Lanese RR 1985 Anabolic steroid use and perceived effects in ten weight-trained women athletes. Journal of the American Medical Association 253 2871-2873.

Tobias JH, Gallagher A \& Chambers TJ 1994 5a-Dihydrotestosterone partially restores cancellous bone volume in osteopenic ovariectomized rats. American Journal of Physiology 267 E853-E859.

Venken K, De Gendt K, Boonen S, Ophoff J, Bouillon R, Swinnen JV, Verhoeven G \& Vanderschueren D 2006 Relative impact of androgen and estrogen receptor activation in the effects of androgens on trabecular and cortical bone in growing male mice: a study in the androgen receptor knockout mouse model. Journal of Bone and Mineral Research 21 576-585.

Vicencio JM, Ibarra C, Estrada M, Chiong M, Soto D, Parra V, Diaz-Araya G, Jaimovich E \& Lavandero S 2006 Testosterone induces an intracellular calcium increase by a nongenomic mechanism in cultured rat cardiac myocytes. Endocrinology 147 1386-1395.

Wakley GK, Schutte HD Jr, Hannon KS \& Turner RT 1991 Androgen treatment prevents loss of cancellous bone in the orchidectomized rat. Journal of Bone and Mineral Research 6 325-330. 
Walters KA, Allan CM, Jimenez M, Lim PR, Davey RA, Zajac JD, Illingworth P \& Handelsman DJ 2007 Female mice haploinsufficient for an inactivated androgen receptor (AR) exhibit age-dependent defects that resemble the AR null phenotype of dysfunctional late follicle development, ovulation, and fertility. Endocrinology 148 3674-3684.

Walters KA, McTavish KJ, Seneviratne MG, Jimenez M, McMahon AC, Allan CM, Salamonsen LA \& Handelsman DJ 2009 Subfertile female androgen receptor knockout mice exhibit defects in neuroendocrine signaling, intraovarian function, and uterine development but not uterine function. Endocrinology 150 3274-3282.

Wronski TJ, Walsh CC \& Ignaszewski LA 1986 Histologic evidence for osteopenia and increased bone turnover in ovariectomized rats. Bone 7 119-123.

Yamazaki I \& Yamaguchi H 1989 Characteristics of an ovariectomized osteopenic rat model. Journal of Bone and Mineral Research 4 13-22.
Yeh S, Tsai MY, Xu Q, Mu XM, Lardy H, Huang KE, Lin H, Yeh SD, Altuwaijri S, Zhou X et al. 2002 Generation and characterization of androgen receptor knockout (ARKO) mice: an in vivo model for the study of androgen functions in selective tissues. PNAS $\mathbf{9 9}$ 13498-13503.

Yeh S, Hu YC, Wang PH, Xie C, Xu Q, Tsai MY, Dong Z, Wang RS, Lee TH \& Chang C 2003 Abnormal mammary gland development and growth retardation in female mice and MCF7 breast cancer cells lacking androgen receptor. Journal of Experimental Medicine 198 1899-1908.

Received in final form 10 March 2010 Accepted 15 April 2010

Made available online as an Accepted Preprint 15 April 2010 